DOI $10.35381 / \mathrm{cm} \cdot v 6 \mathrm{i} 10.207$

\title{
Estilos de aprendizaje en estudiantes superdotados del Ecuador
}

\section{Learning styles in gifted students of Ecuador}

\author{
Zila Esteves Fajardo \\ zilaisabelesteves@hotmail.es \\ Universidad de Guayaquil, Guayaquil \\ Ecuador \\ https://orcid.org/0000-0002-2283-5370 \\ Ramón Bolívar Casquete Muñoz \\ rbcm3177@yahoo.com \\ Universidad Técnica de Babahoyo, Extensión Quevedo \\ Ecuador \\ https://orcid.org/0000-0002-6699-5835 \\ Rosa Elvira Vasconez \\ rosaelviravasconez@hotmail.com \\ Universidad Técnica de Babahoyo, Extensión Quevedo \\ Ecuador \\ https://orcid.org/0000-0001-8150-3758 \\ Miriam Calle Cobos \\ miriam.callec@ug.edu.ec \\ Universidad de Guayaquil, Guayaquil \\ Ecuador \\ https://orcid.org/0000-0002-6699-5835
}

Recibido: 15 de junio de 2019

Aprobado: 1 de agosto de 2019

\section{RESUMEN}

En la actual investigación se analizan los estilos de aprendizaje en estudiantes superdotados del Ecuador, con la finalidad de conocer la preferencia de estilo predominante y articular una orientación sobre el abordaje escolar a desarrollarse con pertinencia de promover una educación inclusiva y de calidad, esto a partir de estudiar el modelo de cuadrantes cerebrales de Ned Herrmann. Se utilizó una metodología descriptiva. Urge fortalecer la perspectiva del aprendizaje centrada en el estudiante, esto 
permitirá que mediante la investigación e indagación, el estudiante genere un aprendizaje en razón de sus exigencias, perspectivas, en posibilidad de crecer en consonancia a los múltiples retos globales.

Descriptores: Aprendizaje visual; Enseñanza y formación; Autoaprendizaje; Estudios por libre.

\section{ABSTRACT}

In the current investigation, the learning styles of gifted students of Ecuador are analyzed, with the purpose of knowing the predominant style preference and articulating an orientation on the school approach to develop with relevance to promote an inclusive and quality education, this from of studying the brain quadrant model of Ned Herrmann. A descriptive methodology was used. It is urgent to strengthen the perspective of studentcentered learning, this will allow the student to generate learning based on their demands, perspectives, in the possibility of growing in line with the multiple global challenges.

Descriptors: Visual learning; Teaching and training; Self instruction; Independent study.

\section{INTRODUCCIÓN}

Los estudiantes superdotados, genios, prodigios, precoces, son algunos de los adjetivos con los cuales se califica a quienes demuestran poseer una inteligencia que sorprende al aprender sobre la media de la población, Rosedo (2019), destaca que "la Asociación Nacional para Niños Superdotados de EE.UU. Ios define como chicos que van 10\% más arriba de la media, para su edad, en lo académico, arte". Esto implica que los estudiantes superdotados aprenden a un ritmo de mayor aceleración que el resto de sus compañeros, lo cual puede implicar que poseen preferencia por un determinado estilo de aprendizaje o quizás por la combinación de estilos, lo cual hace posible que generen un aprendizaje que se considera como superdotado.

Por consiguiente, aprendizaje se comprende como un proceso mediante el cual, la persona puede adquirir conocimientos y emplearlos para realizar actividades en su vida diaria, así Alonso et al., (2012), explican que "el aprendizaje no sólo debe entenderse como un conjunto de conocimientos que se desarrollan en la mente, sino también como 
habilidades que pueden modificarse y mejorarse con el tiempo", esto conlleva a la posibilidad de exponer que el aprendizaje se adquiere desde la diversidad de la persona en preferencia cognitiva, fisiológica, con el medio con el cual interactúa.

Es así que en la actual investigación se analizan los estilos de aprendizaje en estudiantes superdotados del Ecuador, con la finalidad de conocer la preferencia de estilo predominante y articular una orientación sobre el abordaje escolar a desarrollarse con pertinencia de promover una educación inclusiva y de calidad, esto a partir de estudiar el modelo de cuadrantes cerebrales de Ned Herrmann.

\section{REFERENCIAL TEÓRICO}

\section{Modelo cuadrantes cerebrales}

Este modelo ideado por Ned Herrmann, advierte la división del cerebro en cuatro hemisferios, cada lado se caracterizada en forma diferenciada del otro, de ese modo, se puede proceder a aprender de diversos modos en preferencia, según el hemisferio dominante de la persona, en este sentido, (Rojas, 2006), plantea que:

Para Herrmann la dominancia cerebral tiene que ver con la manera de preferencia para aprender, entender y expresar algo, llamado modos preferidos de conocimiento, que es el que se usa con más probabilidad al enfrentarse con la necesidad de resolver un problema o seleccionar una experiencia de aprendizaje.

Esto implica que según la zona de desarrollo del cerebro, se produce un interés por aprender por parte de la personas, Alcalá Flores (2003), cita a De la Parra quien señala: "El desarrollo de la corteza cerebral estimula uno de los cuatro cuadrantes de manera predominante, generando que los individuos tiendan a tener gustos, preferencias, procesamiento mental y esquematización de la personalidad particulares". Es así que Rojas (2006), describe los cuatros hemisferios del siguiente modo:

Cortical izquierdo (Racional). Tiene dificultades para integrar conocimientos a partir de experiencias informales. Prefiere conocer la teoría, el funcionamiento de las cosas antes de pasar a la experimentación. Le gusta las clases sólidas, argumentadas, apoyadas en 
los hechos y las pruebas. Va a clase a aprender, tomar apuntes, avanzar en el programa para conocerlo bien al final del curso.

Comportamiento: frio, distante, pocos gestos, voz elaborada intelectualmente brillante; evalúa, crítica; irónico; le gustan las citas; competitivo; individualista.

Procesos: Análisis; razonamiento; lógica; Rigor, claridad; le gustan los modelos y las teorías; colecciona hechos; procede por hipótesis; le gusta la palabra precisa.

Competencias: Abstracción; matemático; cuantitativo; finanzas; técnico; resolución de problemas. Tiene necesidad de hechos.

Aprende por: razonar a través de ideas, valora el pensamiento lógico, necesita hechos y datos, forma teorías, construye casos. Responde a: lectura formal, discusiones de casos, libros de texto, aprendizaje programado, diseño de modificación de aprendizaje.

Límbico izquierdo (organizado). Se atiene a la organización, le gustan los avances planificados, necesita una clase estructurada para integrar conocimientos y tener el ánimo disponible para ellos. Comportamientos: Introvertido; emotivo, controlado; minucioso, maniático; monologa; le gustan las fórmulas; conservador, fiel; defiende su territorio, ama el poder. Procesos: Planifica; formaliza; estructura; define los procedimientos; ritualista; metódico.

Competencias: Administración; organización; realización, puesta en marcha; conductor de hombres; orador; trabajador consagrado. Metódico, organizado, y frecuentemente meticuloso. Le gusta que la clase se desarrolle según una liturgia conocida y rutinaria. Aprende por: prueba de teoría, estructura, proceso adquisición de habilidades a través de la práctica. Responde a: contextos secuenciales y estructurados, discusiones de caso, aprendizaje programado, diseños de modificación de aprendizaje.

Cortical derecho (Experimental). Necesita apertura y visión de futuro a largo plazo. Aprecia la originalidad, la novedad y los conceptos que hacen pensar. Toma pocas notas porque sabe seleccionar lo esencial. A veces impresiona como un soñador, o de estar desconectado, pero otras sorprende con observaciones inesperadas y proyectos originales. Comportamientos: Original humor; gusto por el riesgo; espacial; simultáneo; 
le gustan las discusiones; futurista; salta de un tema a otro; discurso brillante; independiente. Procesos: Conceptualización; síntesis; globalización; imaginación; intuición; visualización; actúa por asociaciones; integra por medio de imágenes y metáforas. Competencia: Creación; innovación; espíritu de empresa; artista; investigación; visión de futuro. Aprende por: auto descubrimiento, construye conceptos, valora la intuición, busca posibilidades ocultas o no evidentes. Responde a: experiencia, experimental, visual, estético, diseño de aprendizaje individual.

Límbico derecho (sentimental). Se atienen a la comunicación y a la relación. Funciona por el sentimiento y el instinto. Necesita compartir lo que oye para verificar que ha comprendido la lección. Comportamientos: Extravertido; emotivo; espontáneo; gesticulador; lúdico; hablador; idealista, espiritual; busca la aprobación; reacciona mal a las críticas. Procesos: Integra por la experiencia; se mueve por el principio de placer; fuerte implicación afectiva; trabaja con sentimientos; escucha; pregunta; necesidad de compartir; necesidad de armonía; evalúa los comportamientos. Competencias: Relacional; contactos humanos; diálogo; enseñanza; trabajo en equipo; expresión oral y escrita. Trabaja si el profesor es de su gusto; se bloquea y despista fácilmente si no se consideran sus progresos o dificultades. Le gustan algunas materias, detesta otras y lo demuestra. Aprecia las salidas, videos, juegos y todo aquello que no se parezca a una clase. Aprende por: escuchar y compartir ideas, valora el pensamiento intuitivo, trabaja por la armonía del equipo, integra las experiencias al ser. Responde a: actividades y experiencias, que involucren los sentidos, música, interacción de grupos. 


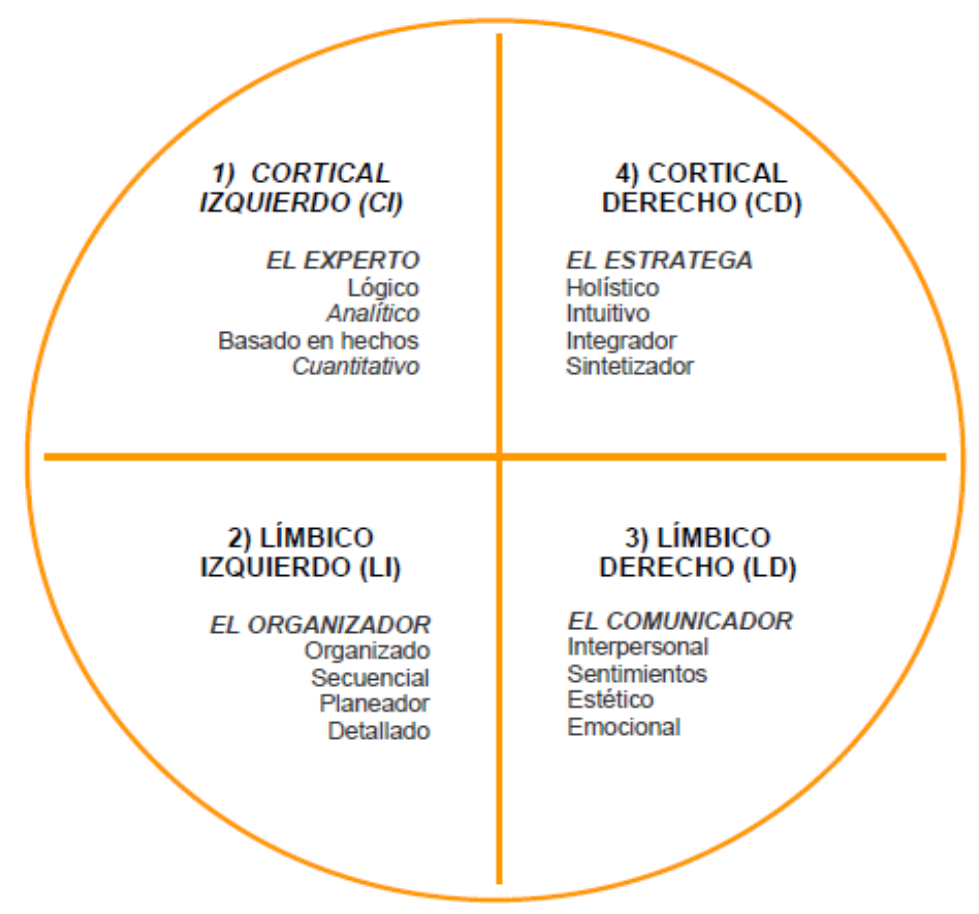

Figura 1. Modelo cuadrantes cerebrales. (De la Parra Paz, 2004).

\section{METODOLOGÍA DE LA INVESTIGACIÓN}

Se aplicó una investigación tipo descriptiva, definida por Arias (2009) como la investigación que "consiste en la caracterización de un hecho, fenómeno o supo con establecer su estructura o comportamiento" (p. 64). Se abordó desde un diseño no experimental, transeccional de campo. Hernández, Fernández, Baptista (2014) refieren al diseño no experimental como "los estudios que se realizan sin la manipulación deliberada de variables y en los que sólo se observan los fenómenos en su ambiente natural para después analizarlos" (p. 267).

La población de acuerdo a Tamayo y Tamayo (2007), "Es la totalidad del fenómeno a estudiar donde las unidades de población poseen una característica común la cual se estudia y da origen a los datos de la investigación" (p. 114), la cantidad de población estuvo conformada 8 estudiantes de instituciones educativas del Ecuador que poseen las características de superdotados por encontrarse por encima de la media promedio de sus 
compañeros en rendimiento académico a quienes se les aplicó el test de Ned Herrmann para conocer el cuadrante predominante en su aprendizaje.

\section{RESULTADOS}

En cuanto a los resultados se tiene que para el cortical izquierdo $(\mathrm{Cl})$ se obtuvo un porcentaje del $37 \%$, para el límbico izquierdo (LI) con un $25 \%$, en cuanto al límbico derecho (LD) un $13 \%$ y para el cortical derecho (CD), un $25 \%$, tal como se refleja en el gráfico 1.

\section{Grafico 1}

Distribución porcentual del modelo de cuadrantes cerebrales

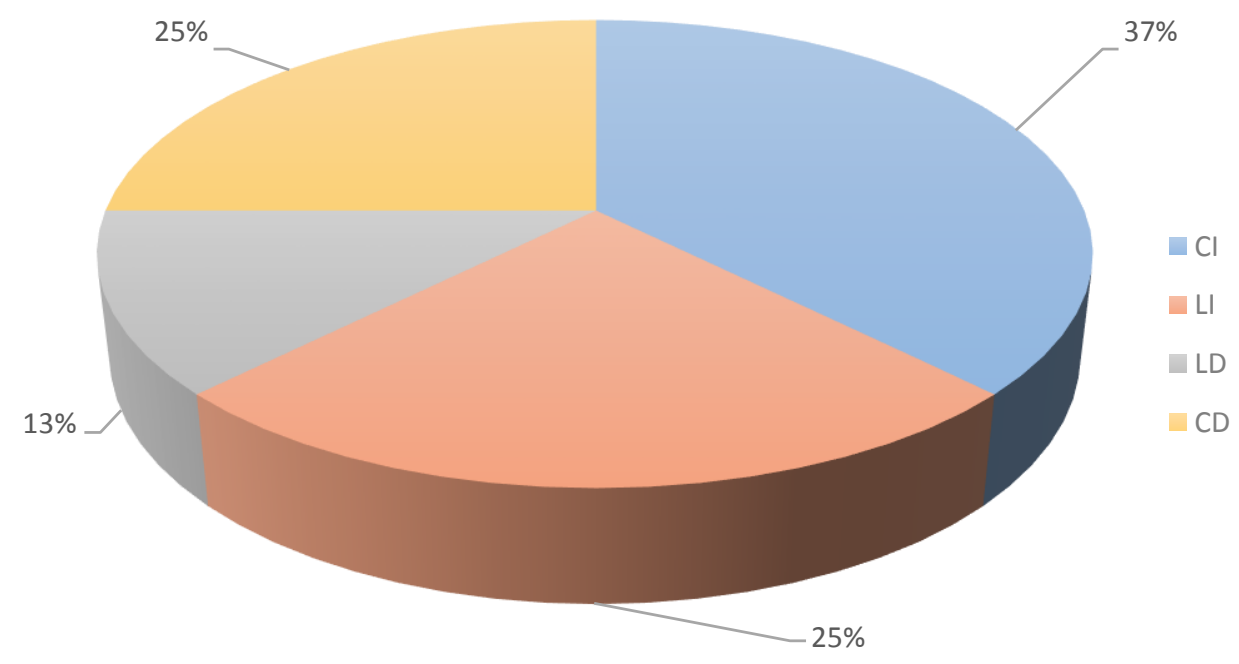

Fuente: Autores a partir de resultados

\section{Discusión}

Los estudiantes con predominancia el cortical izquierdo (Cl), de acuerdo a Ned Herrmann, son personas con un pensamiento o estilo de aprendizaje concreto, analizan la realidad desde evidencias concretas y suelen tener buen humor a partir de la ironía, podrían destacarse en profesiones relacionadas al cálculo matemático, físico, químico, siendo necesario en la escuela fomentar este tipo de conocimientos con la finalidad de promover 
las potencialidades de los estudiantes en aras de promover su vocación profesional en pertinencia a su estilo de aprendizaje.

Los participantes con predominancia en el límbico izquierdo (LI), de acuerdo a Ned Herrmann, se caracterizan por ser planificadores y organizadores, por lo general no desarrollan actividades sin que estas se encuentren planificadas y bajo su supervisión. Este es un estilo de aprendizaje que permite fomentar en los estudiantes la vocación profesional hacia carreras que impliquen ser planificadores, estrategas, proyectándose la posibilidad de promover la inteligencia espacial.

Los estudiantes en relación al límbico derecho (LD), se caracterizan por ser buenos comunicadores y manejadores de las emociones para la movilización de masas, en este sentido, se proyecta un liderazgo carismático transformacional por medio de la motivación, este tipo de estudiantes podrían ser buenos políticos, educadores, líderes religiosos, periodistas, lo cual proyecta que este segmento poblacional se fomenten las competencias pertinentes para promover la vocación laboral en alguna de estas áreas. Quienes se encuentran en el segmento cortical derecho (CD), se caracterizan por tener un aprendizaje visual, creativo e innovador, se destacan profesiones como la arquitectura, diseño, escritores, filósofos, lo cual genera la posibilidad de fomentar un aprendizaje creativo, dinámico, autónomo en los estudiantes, con la finalidad de promover el auto descubrimiento de sus potencialidades.

\section{CONCLUSIONES}

Se acota que con la investigación no se promueve caracterizar la importancia de un estilo de aprendizaje sobre otro, sino de conocerlo como este se desarrolla en estudiantes superdotados con la finalidad de conocer que estrategias de aprendizaje desarrollar para promover un entorno educativo donde el estudiante se vea motivado y favorecido al aprendizaje, esto involucra la posibilidad de germinar una educación inclusiva y de calidad. Así el ministerio de educación del Ecuador (2019), establece que:

La Educación Inclusiva debe ser concebida como un proceso que permite abordar y responder a la diversidad de las necesidades de todos los 
estudiantes a través de una mayor participación en el aprendizaje y reducir la exclusión del sistema educativo. Esto implica cambios y modificaciones de contenidos, enfoques, estructuras y estrategias basados en una visión común y la convicción de que es responsabilidad del Sistema Educativo educar a todos los niños, niñas y adolescentes. El propósito de la Educación Inclusiva es permitir que los docentes y estudiantes se sientan cómodos ante la diversidad y la perciban no como un problema, sino como un desafío y una oportunidad para enriquecer el entorno de aprendizaje.

Los estudiantes superdotados, si no son integrados asertivamente al aprendizaje, pueden verse excluidos, por cuanto un modo de establecer exclusión es obligarles a estudiar temas que ya ellos conocen y dominan, lo cual genera inquietud, así como conductas disruptivas que pueden desmotivar al estudio formal, es necesario propiciar en este tipo de estudiantes, las condiciones de integración al aprendizaje adecuadas, pertinentes, a su realidad, por lo que los cuadrantes de Ned Herrmann, permiten tener una visión clara desde donde se puede focalizar el aprendizaje significativo.

Por otro lado Ramírez (2019), plantea que en el Ecuador la ley obliga a la inclusión, pero no existe formación para abordar con pertinencia esta temática, esto involucra a los superdotados, quienes deberían ser abordados por psicopedagogos especializados en tal fin.

En consecuencia se requiere una educación con visión compleja para atender a todos los estudiantes de la óptica inclusiva y de calidad, Aldana, Salón \& Guzmán (2019), destacan que es necesario promover el pensamiento sistémico para generar interacción entre docentes y estudiantes con la finalidad de generar un abordaje inclusivo del aprendizaje. Peche Cruz \& Giraldo Supo (2019), describen que además urge fortalecer la perspectiva del aprendizaje centrada en el estudiante, esto permitirá que mediante la investigación e indagación, el estudiante genere un aprendizaje en razón de sus exigencias, perspectivas, en posibilidad de crecer en consonancia a las múltiples retos globales. 


\section{REFERENCIAS CONSULTADAS}

1. Aldana, J., Salón, M., \& Guzmán, N. (2019). Liderazgo sistémico en las competencias gerenciales docentes universitarias. CIENCIAMATRIA, 5(8), 50-74. Recuperado a partir de http://cienciamatriarevista.org.ve/index.php/cm/article/view/87

2. Arias, F. (2009). El Proyecto de Investigación. Guía para su elaboración. Caracas: Epísteme. Quinta Edición.

3. Alonso, C., D. Gallego, y P. Honey. (2012). Los estilos de aprendizaje Procedimientos de diagnóstico y mejora (Octava ed.). Bilbao: Mensajero.

4. De la Parra Paz, E. (2004). Herencia de vida para tus hijos. Crecimiento integral con técnicas PNL, Ed. Grijalbo, México.

5. Hernández, Fernández y Baptista (2014). Metodología de la investigación. México, Mc Graw Hill Hispanoamericana. Hill Internacional.

6. Ministerio de educación del Ecuador (2019). Educación inclusiva y especializada. Recuperado de https://educacion.gob.ec/escuelas-inclusivas/

7. Peche Cruz, H., \& Giraldo Supo, V. (2019). El Aprendizaje Flip Learning centrado en el estudiante como generador de calidad educativa. Revista Arbitrada Interdisciplinaria Koinonía, 4(8), 427-450. doi:http://dx.doi.org/10.35381/r.k.v4i8.293

8. Ramírez, S. (2019). Educación inclusiva en Ecuador: hay ley, pero falta formación. Recuperado de https://www.eluniverso.com/noticias/2019/07/23/nota/7438443/educacioninclusiva-ecuador-hay-ley-falta-formacion

9. Rosedo, M. (2019). Hijos superdotados revelan intensidad y sensibilidad. Recuperado de https://www.elcomercio.com/actualidad/hijos-superdotadosrevelan-intensidad-sensibilidad.html

10. Tamayo y Tamayo (2009). El proceso de investigación científica. Limusa. México. 


\section{REFERENCES CONSULTED}

1. Aldana, J., Salón, M., \& Guzmán, N. (2019). Systemic leadership in university teaching management skills. SCIENCIAMATRY, 5 (8), 50-74. Retrieved from http://cienciamatriarevista.org.ve/index.php/cm/article/view/87

2. Arias, F. (2009). The Research Project. Guide for its elaboration. Caracas: Epísteme. Fifth Edition.

3. Alonso, C., D. Gallego, and P. Honey. (2012). Learning styles Diagnostic and improved procedures (Octava ed.). Bilbao: Mensajero.

4. From the Parra Paz, E. (2004). Life inheritance for your friends. Integral growth with NLP techniques, Ed. Grijalbo, Mexico.

5. Hernández, Fernández and Baptista (2014). Research methodology. Mexico, Mc Graw Hill Hispanoamericana. Hill International.

6. Ministry of Education of Ecuador (2019). Inclusive and specialized education. Retrieved from https://educacion.gob.ec/escuelas-inclusivas/

7. Peche Cruz, H., \& Giraldo Supo, V. (2019). The Learning Flip Learning centered on the student as a generator of educational quality. Interdisciplinary Arbitrated Journal Koinonía, 4 (8), 427-450. doi: http: //dx.doi.org/10.35381/r.k.v4i8.293

8. Ramirez, S. (2019). Inclusive education in Ecuador: hay ley, but lack training. Retrieved https://www.eluniverso.com/noticias/2019/07/23/nota/7438443/educacioninclusiva-ecuador-hay-ley-falta-formacion

9. Rosedo, M. (2019). Gifted people reveal intensity and sensitivity. Retrieved from https://www.elcomercio.com/actualidad/hijos-superdotados-revelan-intensidadsensibilidad.html

10. Tamayo and Tamayo (2009). The process of scientific investigation. Limousine Mexico. 